Rev. Int. Contam. Ambie. 35 (4) 1011-1023, 2019

DOI: 10.20937/RICA.2019.35.04.19

\title{
SEPARACIÓN DE RESIDUOS INORGÁNICOS RECICLABLES EN TIJUANA
}

Separation of recyclable inorganic waste in Tijuana

Abisaí ARAGÓN CRUZ ${ }^{1 *}$ y Ana CÓRDOVA ${ }^{2}$

${ }^{1}$ Universidad de la Sierra Sur, Calle Guillermo Rojas Mijangos S/N, Esq. Av. Universidad Col. Ciudad Universitaria, Miahuatlán de Porfirio Díaz, Oaxaca, México C.P. 70800, México

${ }^{2}$ El Colegio de la Frontera Norte, Av. Insurgentes No. 3708, Col. Los Nogales, Cd. Juárez, Chihuahua, México, C.P. 32350, México

*Autor de correspondencia: abisai.aragon.cruz@gmail.com

(Recibido: agosto 2017, aceptado: febrero 2019)

Palabras clave: desechos urbanos, hábitos, motivaciones y dificultades, reciclaje

\section{RESUMEN}

En el marco de la política ambiental de las 3R (reducir, reutilizar y reciclar) y de la valoración de los residuos sólidos urbanos (RSU), la separación desde la fuente de origen se ha planteado como una estrategia para disminuir los efectos negativos del incremento de la generación de dichos residuos. A partir de un enfoque participativo, actores gubernamentales y no gubernamentales han buscado que los individuos pongan en práctica formas de comportamiento sustentables desde el hogar, sin el efecto esperado. Dado los diversos hábitos de separación, el presente estudio tiene el objetivo de identificar y explicar los factores que afectan o determinan el comportamiento de dicha práctica en los habitantes de Tijuana, específicamente en la separación de los residuos sólidos inorgánicos reciclables. Para ello, se analizaron las motivaciones y las dificultades reportadas por los miembros de las viviendas. La información se obtuvo a través de la aplicación de una encuesta distribuida en 44 áreas geoestadísticas básicas de la localidad de Tijuana. Los principales hallazgos muestran que $56.6 \%$ de los encuestados reportó separar sus residuos generados; esto con mayor frecuencia en las viviendas con un grado de marginación alto y medio, $69 \%$ y $59.1 \%$, respectivamente. La motivación más reportada se relaciona con el cuidado al ambiente, y que el camión recolector mezcle los residuos reciclables con el resto de los desechos sólidos, fue la dificultad más mencionada. Este estudio complementa a otras investigaciones sobre el tema y aporta un acercamiento explicativo de los hábitos de separación en las viviendas.

Key words: urban waste, habits, motivations and difficulties, recycling

\begin{abstract}
Within the framework of the $3 \mathrm{R}$ environmental policy (reduce, reuse and recycle) and valuation of urban solid waste (USW), source separation has been presented as one strategy to reduce the negative effects of increasing amounts of USW. From a participative approach, government and non-government actors have sought to encourage people to implement sustainable behavior from their homes, without the expected effect. Given the diversity in household customs, this study seeks to identify and explain the factors that affect or determine the behavior of this practice in the residents of Tijuana,
\end{abstract}


specifically in the separation of their recyclable inorganic solid waste. The variables that were analyzed are the motivations and the difficulties reported by the householders. To collect the data a survey was distributed within 44 basic geo-statistical areas in the Tijuana locality. The findings expressed that $56.6 \%$ of survey respondents reported to separate their waste; this behavior was common in homes with a high and medium scale of marginality, $69 \%$ and $59.1 \%$, respectively. The most mentioned incentive is connected to caring for the environment, and the most reported barriers were the mixing of recyclable products with the rest of the solid waste. This study complements previous research on this topic and provides an explanation of separation habits, specifically at the household level.

\section{INTRODUCCIÓN}

El volumen de los residuos sólidos urbanos (RSU) en México ha tenido un aumento significativo desde 1950, convirtiéndose en un problema para las ciudades que generan desechos sin tener una gestión adecuada de los mismos. Esta situación impacta ambiental y socialmente cada vez más, pues el sistema natural tiene una capacidad limitada de absorberlos o procesarlos, sin verse afectado.

El incremento puede explicarse por factores demográficos, sociales, económicos y culturales. Los patrones de consumo, modificaciones tecnológicas, demanda de bienes y servicios, procesos de industrialización y el crecimiento poblacional son algunas causas que inciden en el escenario actual y muestran la complejidad del problema (Izazola 2014).

Ante esta coyuntura y ante numerosas alternativas de solución, diversos actores públicos, privados y sociales buscan motivar a los individuos a poner en práctica formas de comportamiento sustentables, principalmente la separación de los residuos generados en las casas habitación que resultan de actividades domésticas, para que sean enviados a procesos de reciclaje.

Esta opción ha sido abordada desde la política de las 3R (reducir, reutilizar y reciclar), y más aún por la valorización de los residuos (CDHCU 2003); principio contemplado en la ley general para la prevención y gestión integral de los residuos (LGPGIR), que si bien ha comenzado a implementarse, aún falta por transitar de "la participación de [...] un mero almacenamiento temporal de los residuos [...] a una participación activa y corresponsable" (Rodríguez y Córdova 2006, Rodríguez 2008, Mercado 2014). "Mediante el reciclaje, los productos pueden volver a manufacturarse sin tanta necesidad de materiales vírgenes, mitigando así la presión sobre el acervo de recursos" (Mercado 2014). Aprovechar los residuos como insumos en las actividades productivas requie- re, como primer paso, separar los RSU susceptibles a ser valorizados.

En el marco de las $3 \mathrm{R}$ y la valorización de los residuos, en Tijuana actores privados, públicos y sociales han planteado acciones, que van desde la construcción a nivel municipal de una planta separadora de RSU en 2008 (misma que dejó de funcionar en 2012 principalmente por la variabilidad en los precios de los materiales reciclables y la insuficiente cantidad de los residuos procesados), hasta diversas iniciativas de gestión de residuos (centro de compostaje urbano, campañas educativas de concientización ambiental y de limpieza, programa piloto de separación de residuos orgánicos domésticos, y programas de acopio y reciclaje) promovidas por organizaciones no gubernamentales (Couto 2008, UABC y MKC 2012).

La separación desde el origen, a nivel doméstico, necesariamente precisa de la participación de la población y la práctica ha demostrado lo difícil que es encontrar una forma adecuada de incentivar la colaboración de las personas en el manejo de los residuos. Hay una serie de factores que inciden positivamente y otros que merman en lo anterior; lo primero es resultado de estímulos percibidos, mientras que lo segundo es consecuencia de las barreras observadas (Garcés et al. 2002, Palatnik et al. 2005, Aprilia et al. 2011, Mercado 2014).

En el cuadro I se resumen los factores identificados en la literatura que incentivan y desincentivan la separación de reciclables desde el hogar. La disposición a separar también es afectada por variables sociodemográficas, como los ingresos anuales, la edad, el género, el tipo de empleo, la educación y el lugar de residencia (Garcés et al. 2002).

Visto de esta manera, en un contexto donde interactúan diversos actores gubernamentales y no gubernamentales, la búsqueda de soluciones es más compleja. La pluralidad de actores hace que las propuestas basadas en la participación social sean difíciles de consensuar, sin embargo son una oportu- 
CUADRO I. FACTORES QUE INCIDEN EN LA SEPARACIÓN DOMÉSTICA DE RESIDUOS RECICLABLES

\begin{tabular}{|c|c|}
\hline Incentivos & Desincentivos \\
\hline 1. Conocimiento/conciencia ambiental & Esfuerzo que requiere la separación de RSU \\
\hline $\begin{array}{l}\text { 2. Percepción positiva de la gestión municipal en el } \\
\text { manejo de RSU }\end{array}$ & $\begin{array}{l}\text { 2. Falta de tiempo por las responsabilidades en el hogar y el } \\
\text { cuidado de los hijos }\end{array}$ \\
\hline 3. Posibilidades de ganancias económicas & Falta de espacio en el hogar para almacenar los residuos \\
\hline 4. Oportunidades de empleo & Falta de contenedores para residuos separados (domésti- \\
\hline 5. Oportunidades de desarrollo social & cos o municipales) y su uso inadecuado \\
\hline 6. Disposición general a colaborar en la comunidad & $\begin{array}{l}\text { 5. Falta de un sistema de recolección municipal de residuos } \\
\text { separados }\end{array}$ \\
\hline & 6. Distancia entre el domicilio y el centro o lugar de acopio \\
\hline & Mercado de reciclaje limitado \\
\hline
\end{tabular}

RSU $=$ residuos sólidos urbanos. Fuente: Elaboración propia con información de Aprilia et al. 2011, Shahjahan 2010, Davies 2007, Garcés et al. 2002

nidad de crear redes de colaboración en gobernanza, haciendo que la gestión y el manejo de los desechos urbanos sea sinérgica (Kütting 2004, Palmujoki 2006, Valderrábano et al. 2011).

Bajo esta línea de análisis y dada la vasta literatura respecto al funcionamiento técnico, operativo y organizacional de la gestión integral de los residuos sólidos urbanos (GIRSU), la presente investigación se enfocó a estudiar los factores que inciden en la separación de los residuos sólidos inorgánicos reciclables (papel, plástico, metal y vidrio) generados en las casas habitación, que en el resto del documento se denominarán "residuos reciclables". Cabe aclarar que no fueron considerados los residuos con características domiciliarias provenientes de cualquier otra actividad dentro de establecimientos o en la vía pública, ni los residuos generados por actividades de limpieza de las vías y lugares públicos, que conforme a la LGPGIR (art. 5, fracc. XXXIII), también son considerados como RSU. El estudio complementa a otras investigaciones sobre el manejo de residuos en Tijuana y en México y además aporta elementos para entender cuál ha sido la respuesta de los habitantes ante el escenario descrito.

\section{MATERIALES Y MÉTODOS}

Con el objetivo de conocer la disposición a separar y analizar las razones así como las características sociodemográficas que explican los hábitos de separación domiciliaria de los residuos reciclables, se aplicó como técnica de investigación una encuesta "cara a cara" compuesta por 350 cuestionarios (instrumento de medición); cada uno estructurado por 36 ítems y organizado en cinco secciones. El cuestionario diseñado para este estudio permitió examinar numéricamente reportes verbales, teniendo en cuenta que puede haber diferencias entre lo reportado por cada individuo y su comportamiento real.

La encuesta se aplicó en la localidad de Tijuana (una de las 16 áreas geográficas que existen en el municipio del mismo nombre), conformada por 438 801 viviendas (lo que representa un $84.6 \%$ de un total de 518191 viviendas registradas) ubicadas en 455 áreas geoestadísticas básicas urbanas (AGEB-U) de un total de 573 a nivel municipal (INEGI 2010, SCINCE 2012, IMPLAN 2014).

Para incluir las características sociodemográficas de la zona de estudio, la localidad se estratificó con base en el índice de marginación urbana (IMU) del Consejo Nacional de Población (CONAPO), que contempla cinco estratos o grados de marginación: muy alto, alto, medio, bajo y muy bajo; el primero denota la mayor carencia en la capacidad de adquisición de bienes y servicios (educación, salud y vivienda) como puede observarse en el cuadro II.

El tamaño de la muestra se determinó con la siguiente fórmula (Manzano 1998) considerando como población objetivo el total de viviendas (438 801) de la localidad:

$\left.n=\left\{\left(Z^{2}(p X q) / e^{2}\right)\right\} /\left\{1+\left[\left(Z^{2}(p X q) / e^{2}\right)-1\right)\right] / N\right\}$

Dónde:

$Z($ nivel de confianza $)=1.64$

$e($ error de muestreo $)=0.05$

$p$ y $q$ (varianza de la población $)=0.5$

$N($ tamaño del universo $)=438801$ viviendas

Al realizar la sustitución y con un valor esperado del $15 \%$ en pérdidas se obtuvo un resultado de $n=$ 318 viviendas a encuestar.

Para validar los instrumentos de medición se realizó una prueba piloto con fines exploratorios 
CUADRO II. CARACTERÍSTICAS SOCIODEMOGRÁFICAS DE LAS ÁREAS GEOESTADÍSTICAS BÁSICAS URBANAS

\begin{tabular}{|c|c|c|c|c|c|c|c|c|c|c|}
\hline $\begin{array}{l}\text { Marginación } \\
\text { urbana }\end{array}$ & Clave & Población & Ind 1 & Ind2 & Ind3 & Ind4 & Ind5 & Ind6 & Ind7 & Ind8 \\
\hline Muy alto & 6614 & 3613 & 8 & 59.6 & 31 & 42.9 & 50 & 19.9 & 50.2 & 17.1 \\
\hline \multirow[t]{6}{*}{ Alto } & 1810 & 2752 & 9.8 & 56.6 & 42.3 & 33.9 & 35.8 & 10.8 & 43 & 10.7 \\
\hline & 4410 & 4558 & 8.9 & 54.5 & 41.2 & 19.1 & 3.1 & 6.3 & 40.2 & 14.7 \\
\hline & 5245 & 3143 & 5.1 & 50.7 & 37.3 & 17 & 12.3 & 8.4 & 43.8 & 11.9 \\
\hline & 5372 & 2739 & 10.6 & 53.4 & 42.4 & 18.7 & 2.8 & 9 & 43.1 & 13.6 \\
\hline & 5902 & 5239 & 4.1 & 51.2 & 40.4 & 25.9 & 5.2 & 10.4 & 47.1 & 7.9 \\
\hline & 6648 & 156 & 12.1 & 57.2 & 25.4 & 68.7 & 3.1 & 9.3 & 46.8 & 6.2 \\
\hline \multirow[t]{16}{*}{ Medio } & 1492 & 2807 & 4.4 & 32.2 & 40.5 & 1.9 & 0.3 & 0.8 & 19.8 & 9.3 \\
\hline & 1774 & 2269 & 4.7 & 34 & 38.9 & 1.7 & 0.3 & 0.3 & 18.8 & 5.2 \\
\hline & 1789 & 2091 & 7.5 & 45.1 & 34.7 & 6.1 & 0.7 & 2.3 & 29.4 & 8.9 \\
\hline & 1882 & 4463 & 5.7 & 46.9 & 34.1 & 15.7 & 1.2 & 6.5 & 37.4 & 7.6 \\
\hline & 1948 & 7179 & 5.2 & 49.5 & 30.3 & 10.1 & 2.8 & 9.1 & 42.5 & 10.4 \\
\hline & 2927 & 5383 & 3.7 & 34.3 & 45.6 & 7.2 & 0.3 & 0.2 & 21.6 & 12.9 \\
\hline & 3075 & 5315 & 4.2 & 38.7 & 45.9 & 2.2 & 0.1 & 1.1 & 24.3 & 6.4 \\
\hline & $308 \mathrm{~A}$ & 3592 & 4.4 & 33.7 & 47.2 & 1.5 & 0.1 & 0.6 & 23.8 & 5.7 \\
\hline & 3179 & 3186 & 3.5 & 55.4 & 45 & 13.3 & 4.8 & 4.9 & 32.8 & 8.7 \\
\hline & 3501 & 5173 & 2.9 & 34.4 & 38.3 & 9.1 & 7.4 & 2.3 & 20.9 & 6.5 \\
\hline & 3874 & 5120 & 6.2 & 38.9 & 35.9 & 8.9 & 0.2 & 0.7 & 24.6 & 14.1 \\
\hline & 4213 & 2199 & 4.3 & 43.5 & 37 & 14.2 & 5.4 & 1.9 & 24.7 & 8.4 \\
\hline & $429 \mathrm{~A}$ & 2907 & 5 & 39.5 & 35.4 & 7.2 & 0.2 & 6.3 & 34.4 & 5.5 \\
\hline & 5508 & 1956 & 2.9 & 50.1 & 33.7 & 25.3 & 3.3 & 9.2 & 43.7 & 6.8 \\
\hline & 6489 & 5459 & 2.9 & 26.3 & 25.4 & 5.6 & 2.4 & 7.4 & 32.4 & 2.7 \\
\hline & 7167 & 3642 & 5.3 & 44.4 & 39.6 & 21.8 & 5.3 & 5.4 & 35.6 & 7.5 \\
\hline \multirow[t]{12}{*}{ Bajo } & 2414 & 951 & 5 & 29.5 & 48.1 & 1.8 & 2.1 & 1.8 & 21.3 & 3.6 \\
\hline & 2490 & 1573 & 3.6 & 24.3 & 37.4 & 4.3 & 0.9 & 4.7 & 17.9 & 2.7 \\
\hline & 2965 & 1356 & 1.9 & 37.8 & 33.7 & 0 & 0 & 0 & 15 & 1.8 \\
\hline & 3018 & 3030 & 1.9 & 31.1 & 41.7 & 1.7 & 0.5 & 0.2 & 21.4 & 4.2 \\
\hline & $322 \mathrm{~A}$ & 2972 & 3.9 & 35.8 & 52.6 & 0.4 & 0.1 & 1.1 & 20.4 & 4.7 \\
\hline & 3713 & 1320 & 3.7 & 29.3 & 37.9 & 1 & 0.2 & 5.5 & 16.7 & 2.3 \\
\hline & 3855 & 4082 & 4.6 & 24.6 & 40.5 & 0.9 & 0 & 1.2 & 16.7 & 2.8 \\
\hline & 4660 & 3310 & 4.3 & 24 & 29.2 & 1.8 & 1.4 & 0.2 & 22.1 & 2.3 \\
\hline & 5531 & 3038 & 3.8 & 23.9 & 28.5 & 0.2 & 0 & 0.1 & 24.4 & 2.1 \\
\hline & 6474 & 4865 & 2.8 & 25 & 19.9 & 0 & 0 & 1.8 & 32.9 & 2.5 \\
\hline & 6525 & 5526 & 2.5 & 25.2 & 28.6 & 2.7 & 0.7 & 1.6 & 35.7 & 4.6 \\
\hline & 6629 & 937 & 2 & 26.6 & 15.6 & 0.3 & 0.3 & 0 & 24.6 & 1.1 \\
\hline \multirow[t]{9}{*}{ Muy bajo } & 437 & 2840 & 2.2 & 18.1 & 34.4 & 1.92 & 0 & 2.1 & 8.1 & 1.6 \\
\hline & 441 & 2867 & 1 & 9.1 & 30 & 0 & 0 & 2.8 & 2.9 & 0.3 \\
\hline & 3412 & 3056 & 0.9 & 12.8 & 34.9 & 0.09 & 0 & 2.1 & 5.7 & 0.6 \\
\hline & 3427 & 4126 & 2.9 & 19.1 & 33.9 & 0.2 & 0 & 4 & 8 & 1.6 \\
\hline & 3889 & 3855 & 1.4 & 12 & 34.3 & 0.8 & 0.08 & 0.7 & 9.6 & 1.4 \\
\hline & 5601 & 3525 & 0.3 & 7.4 & 27.4 & 0 & 0 & 1.7 & 1.5 & 0.2 \\
\hline & 5669 & 212 & 0 & 25.1 & 27.3 & 0 & 0 & 0 & 13.8 & 4.6 \\
\hline & $710 \mathrm{~A}$ & 3199 & 1.6 & 22.1 & 33.8 & 0.4 & 0.4 & 0.9 & 13.9 & 2.1 \\
\hline & 7862 & 142 & 0 & 22.222 & 21.9858 & 0 & 0 & 0 & 33.33 & 0 \\
\hline
\end{tabular}

Ind $1=\%$ población de 6 a 14 años que no asiste a la escuela, Ind $2=\%$ población de 15 años o más sin secundaria completa, Ind3 = población sin derecho-habiencia a los servicios de salud, Ind $4=\%$ viviendas particulares sin agua entubada dentro de la vivienda, Ind $5=\%$ viviendas particulares sin drenaje conectado a la red pública o fosa séptica, Ind $6=\%$ viviendas particulares con piso de tierra, Ind $7=\%$ viviendas particulares con algún nivel de hacinamiento, Ind $8=\%$ viviendas particulares sin refrigerador

Fuente: Elaboración propia a partir de datos del CONAPO 2010 

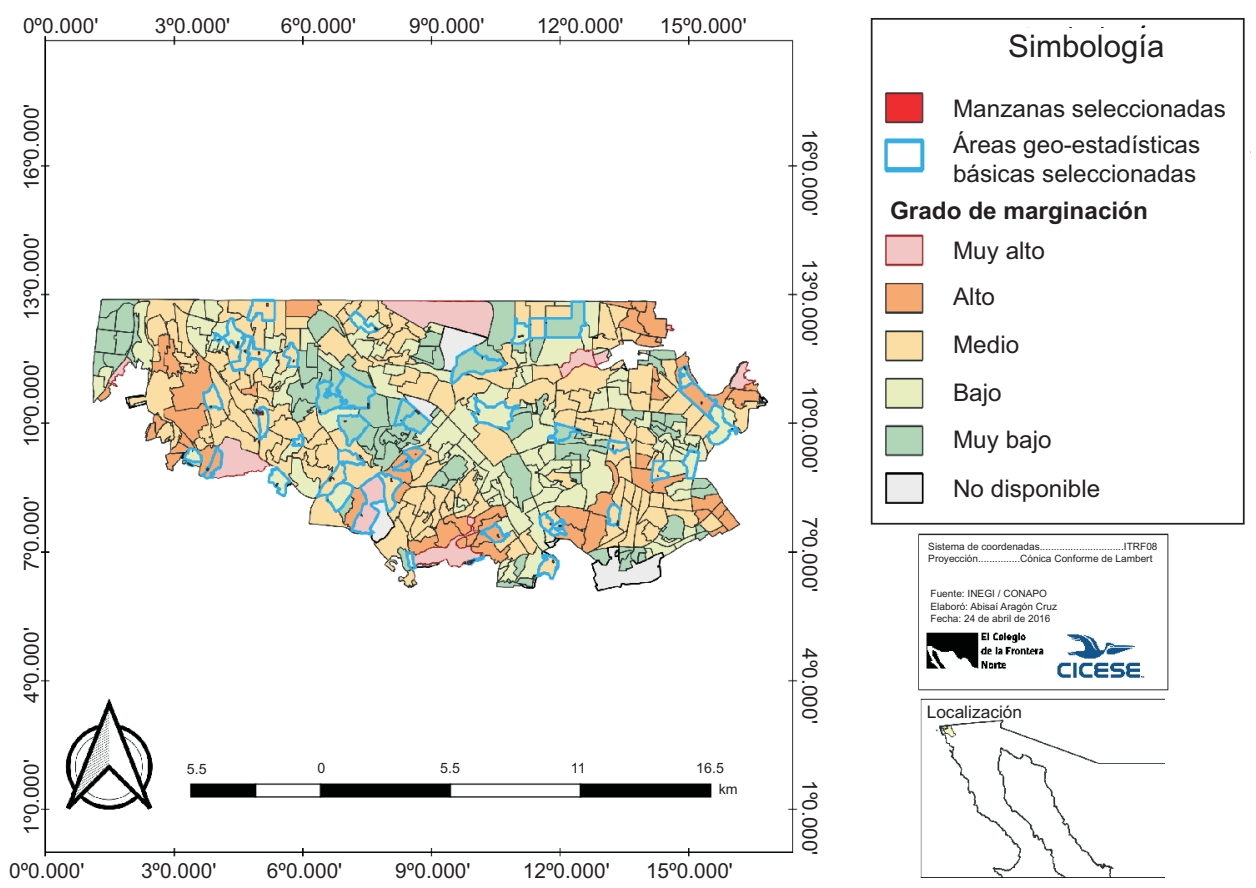

Fig. 1. Zonas de aplicación de la encuesta definitiva Fuente: Elaboración propia con datos del CONAPO 2010, INEGI 2010, SCINCE 2012 y el IMPLAN 2014

y de logística en noviembre de 2015. Se aplicaron 80 cuestionarios distribuidos proporcionalmente en cinco AGEB-U (de distinto estrato) de la localidad; las cuales fueron seleccionadas por conveniencia en una misma zona de estudio. Una vez efectuados los cambios al instrumento de medición se aplicó la encuesta, para lo cual primeramente y con ayuda del programa SPSSC versión 22 se eligió de forma aleatoria al $10 \%$ del total de AGEB-U en cada IMU. Una vez elegidos (Fig. 1), para determinar la asignación y distribución de fracciones del tamaño de la muestra se utilizó la fórmula:

$k s h=n \mathrm{~N}$
Dónde:

$k s h=$ desviación estándar

$n=$ tamaño muestral

$\mathrm{N}=$ tamaño del universo poblacional

Sustituyendo los valores, el factor resultante fue de 0.000724702 , que se aplicó conforme al número de viviendas de cada AGEB-U (Cuadro III).

Posterior a ello se seleccionaron aleatoriamente los puntos de partida (constituidos por manzanas al interior de las AGEB-U previamente elegidas) que pueden observarse en la figura 1 en color rojo. Una vez ubicados y para la aplicación de los cuestionarios, las viviendas (como unidad de análisis) se eligieron

\section{CUADRO III. CUESTIONARIOS POR ÁREA GEOESTADÍSTICA BÁSICA URBANA}

\begin{tabular}{|c|c|c|c|c|c|c|}
\hline $\begin{array}{l}\text { Marginación } \\
\text { urbana }\end{array}$ & $\begin{array}{c}\text { \# de } \\
\text { AGEB-U }\end{array}$ & $\begin{array}{c}\text { \# de } \\
\text { viviendas }\end{array}$ & $\begin{array}{l}\text { \# de AGEB-U visitadas } \\
\text { (10\% de la muestra) }\end{array}$ & $\begin{array}{c}(k s h) \\
\text { (núm. de viviendas) }\end{array}$ & $\begin{array}{c}\text { \# de cuestionarios } \\
\text { programados }\end{array}$ & $\begin{array}{c}\text { \# de cuestionarios } \\
\text { aplicados }\end{array}$ \\
\hline Muy alta & 9 & 5932 & 1 & $(0.000724702)(5,932)$ & 4 & 6 \\
\hline Alta & 61 & 47344 & 6 & $(0.000724702)(47,344)$ & 34 & 39 \\
\hline Media & 164 & 192491 & 16 & $(0.000724702)(192,491)$ & 140 & 154 \\
\hline Baja & 117 & 113983 & 12 & $(0.000724702)(113,983)$ & 83 & 90 \\
\hline Muy baja & 86 & 79051 & 9 & $(0.000724702)(79,051)$ & 57 & 61 \\
\hline Total & $437^{*}$ & 438801 & 44 & No aplica & 318 & 350 \\
\hline
\end{tabular}

AGEB-U = área geo-estadística básica urbana. Fuente: Elaboración propia

*De las 455 AGEB-U de la localidad de Tijuana sólo existe información disponible para 437 
bajo el procedimiento zig-zag (izquierda-derecha) con un salto sistemático de tres viviendas entre vivienda encuestada. Esta etapa se efectuó durante febrero y marzo de 2016 con un total de 350 cuestionarios aplicados.

En la parte operativa, las zonas se ubicaron con ayuda del sistema de información geográfica (SIG) ArcMap(C) versión 10.4 y la plataforma de Google Maps $\subseteq$. La captura de los datos se realizó con la aplicación InfoPath para construir una matriz de datos en Excel y así efectuar un análisis estadístico en el programa SPSSC. Se analizó primero la estadística descriptiva (distribución de frecuencias, medidas de tendencia central y variabilidad de los datos numéricos). Posteriormente, utilizando un modelo de regresión logística binaria $(y=1 \mid x i)$ se efectuó un análisis multivariado para predecir la probabilidad de que la separación de residuos reciclables $(y=1)$ sucediera dada la presencia de diversos factores $(x i)$.

Así pues, la variable dependiente $(y)$ se definió como la presencia (1) o ausencia (0) de miembros de la vivienda que separan los residuos sin importar la periodicidad de su hábito, ni la cantidad de residuos separados. Las $k$ variables explicativas en el modelo estadístico (independientes y de control designadas por $x 1, x 2, x 3, \ldots, x k$ ) identificadas con base en la literatura y en la prueba piloto fueron: el conocimiento de problemas derivados del manejo inadecuado de los residuos, la conciencia sobre la importancia de la separación, la participación en organizaciones o programas de reciclaje, el interés de recibir una capacitación o información relacionada al tema de los residuos y el conocimiento respecto a los centros de acopio de residuos reciclables.
Además, se incorporaron variables socioeconómicas (género, escolaridad, años de residencia en la ciudad y edad) del (a) jefe de familia. A nivel vivienda, se consideró como variable independiente el IMU. Asimismo, se construyó un índice socioeconómico compuesto por tres indicadores: tipo de material de piso predominante, número de focos y los servicios o artefactos electrónicos y electrodomésticos disponibles en la vivienda. Para mayores detalles sobre la estructura del instrumento de medición (cuestionario), la técnica (encuesta) y otros aspectos del diseño de la investigación consultar Aragón (2016).

\section{RESULTADOS}

Los resultados tienen como base 350 cuestionarios distribuidos entre las AGEB-U según se muestra en el cuadro III. Los principales hallazgos del análisis descriptivo respecto a la separación actual reportada de residuos reciclables, mostraron que $63.4 \%(\mathrm{n}=$ 222) de los encuestados realizaron alguna vez algún tipo de separación en su vivienda, esto sin considerar la cantidad de desechos separados, ni la regularidad del hábito.

La razón principal para separarlos tuvo el objetivo de venderlos $(47.3 \%)$, seguido de cerca por el deseo de que alguien los recupere de la vía pública (43.7\%), tal como se observa en la figura 2 . De los que alguna vez realizaron la separación $(\mathrm{n}=222), 10.8 \%(\mathrm{n}$ $=24)$ dejó de hacerlo, por lo que de este total $(\mathrm{n}=$ $222)$, el $89.2 \%(n=198)$ reportó que seguía efectuando la práctica. En números absolutos, de los 350 encuestados, $56.6 \%(\mathrm{n}=198)$ reportó separar los

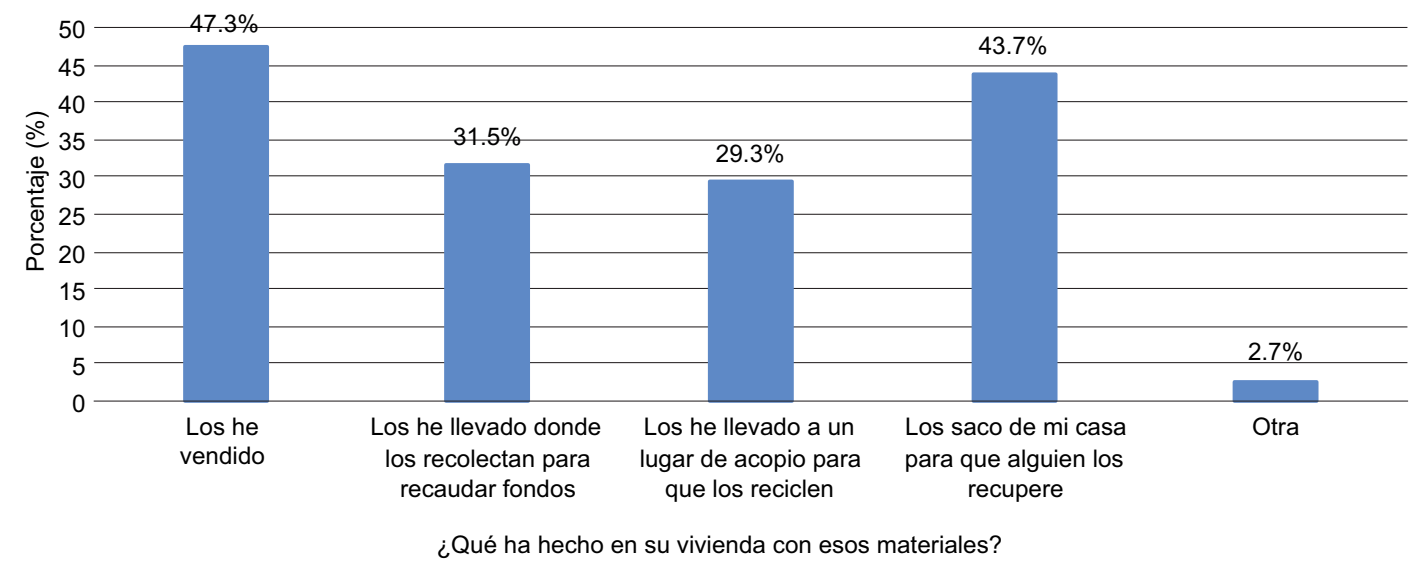

Fig. 2. Manejo de materiales reciclables separados en las viviendas*

*Las respuestas no son mutuamente excluyentes, por lo que la suma es más de $100 \%$ Fuente: Elaboración propia 
residuos reciclables en el momento de la encuesta, esto sin tomar en cuenta como ya se mencionó, la cantidad de los desechos, ni la periodicidad de la acción.

Con estos datos se identificó que existen al menos tres tipos de escenarios en las viviendas en cuanto a los hábitos de separación reportados: 1) Los que nunca han llevado a cabo esta práctica $(36.6 \%, \mathrm{n}=$ $128), 2)$ los que por determinadas razones dejaron de hacerlo $(6.9 \%, \mathrm{n}=24)$ y 3$)$ los que en ese momento conservaban la práctica de separación $(56.6 \%, \mathrm{n}$ $=198$ ). El hábito de separación fue reportado con mayor frecuencia en las viviendas con un grado de marginación alto y medio, $69 \%(\mathrm{n}=20)$ y $59.1 \%$ $(\mathrm{n}=91)$ respectivamente.

En relación al tipo de residuos separados, las viviendas donde se reportó la separación $(56.6 \%, \mathrm{n}=$ 198), el aluminio y el plástico fueron los materiales que se segregaban en mayor cantidad. En menor medida se separaban los demás residuos, tal como se muestra en la figura 3.

En lo que se refiere a los factores que inciden en la separación de los residuos reciclables, se encontraron diversas motivaciones y dificultades entre los

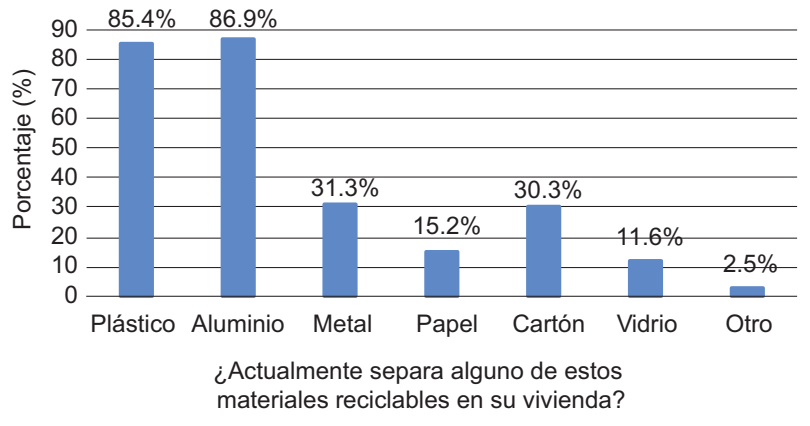

Fig. 3. Materiales separados en las viviendas* *Las respuestas no son mutuamente excluyentes, por lo que la suma es más de $100 \%$

Fuente: Elaboración propia

separadores (S) -encuestados que reportaron realizar actualmente la separación de sus residuos reciclablesy los no separadores (NS) -encuestados que reportan por alguna razón no realizar o dejar de efectuar esta práctica-, que se muestran en el cuadro IV. La motivación con mayor frecuencia para ambos grupos $(81.3 \%$ de $\mathrm{S}$ y $73 \%$ de NS) se relaciona con el cuidado del ambiente.

CUADRO IV. MOTIVACIONES Y DIFICULTADES PARA LLEVAR A CABO LA SEPARACIÓN*

\begin{tabular}{lcc}
\hline \multicolumn{2}{c}{ Motivaciones para llevar a cabo la separación } & \\
\hline & Separadores (\%) & No separadores (\%) \\
\hline Que sé/me enseñaran a separarlos correctamente & 44.9 & 44.7 \\
Que todos en mi comunidad lo hacen/hicieran & 28.3 & 63.2 \\
Conozco/ conocer los lugares donde llevarlos & 43.4 & 63.2 \\
Que una empresa o persona recoja/ recogiera esos materiales & 37.9 & 62.5 \\
Generar empleo a personas dedicadas al reciclaje & 53.0 & 59.9 \\
Cuidar el ambiente & 81.3 & 73.0 \\
Que me paguen/ pagaran por los materiales & 46.5 & 38.8 \\
Poner el ejemplo a los demás & 60.6 & 59.2 \\
Que es/fuera obligatorio & 22.7 & 45.4 \\
\hline & & No separadores \\
\hline \multicolumn{1}{c}{ Dificultades para llevar a cabo la separación } & 40.1 \\
\hline No tengo tiempo para separar & Separadores & 39.5 \\
No tengo tiempo para llevarlos a un punto de acopio & 15.7 & 31.6 \\
No tengo espacio & 17.7 & 41.4 \\
No tengo botes extras & 16.7 & 29.6 \\
No sé dónde llevarlos & 16.7 & 30.3 \\
Genero poca basura para separarlos & 13.1 & 46.1 \\
El camión recolector los vuelve a mezclar con la basura & 14.6 & 40.1 \\
No hay un lugar práctico para llevarlos & 18.2 & 16.4 \\
No sé cuáles materiales son reciclables & 15.2 & 17.1 \\
\hline
\end{tabular}

* Las respuestas no son mutuamente excluyentes, por lo que la suma es más de $100 \%$ Fuente: Elaboración propia 
Para los S, la segunda motivación identificada estuvo relacionada con poner el ejemplo a los demás (60.6 $\%, \mathrm{n}=120)$. En cambio para los NS, las motivaciones que aparecen en segundo lugar fueron "que todos en su comunidad lo hicieran" y "conocer los lugares a donde se podían llevar los reciclables" (ambos con $63.2 \%$, n =96). Llama la atención que tanto para $\mathrm{S}$ como para NS las motivaciones que ocupan el segundo lugar están relacionadas con la participación y normas sociales. Para los NS, además se encuentra la necesidad de que haya alguien que recoja los materiales separados (62.5 $\%, \mathrm{n}=95)$. Para ambos grupos es valioso generar empleo a personas dedicadas al reciclaje $(53 \%, \mathrm{n}=$ 105 y $59.9 \%, \mathrm{n}=91$ respectivamente).

En lo referente a las dificultades, que el camión recolector mezcle los residuos reciclables al momento de su recolección con el resto de los desechos sólidos fue la principal razón que desmotiva tanto a S (18\%) como a NS (46\%). Así pues, la deficiencia en el servicio público de recolección es el principal obstáculo para la separación según los reportes de los encuestados (de las 333 viviendas que cuentan con el servicio, $94.3 \%$ lo recibe de la autoridad municipal, $5.1 \%$ de una empresa o particular, y el resto $(0.6 \%)$ desconoce quién le presta el servicio).

Otras dificultades incluyen la falta de recipientes extra, espacio o tiempo para esta actividad. Tanto para $\mathrm{S}$ como para NS, dificultades menos mencionadas incluyeron "no saber a dónde llevar los residuos separados" (con $13.1 \%$ y $29.6 \%$, respectivamente) o no saber cuáles materiales son reciclables $(6.1 \%$ y $16.4 \%$, respectivamente).

Si bien es cierto que la separación de los residuos reciclables tiene diversos propósitos, es de señalarse que la venta de estos materiales disminuye conforme el grado de marginación es menor. De $47.3 \%(n=$ 105) de los encuestados que reportaron vender sus residuos, el mayor porcentaje se presentó en las zonas con grado de marginación muy alto y alto, con $80 \mathrm{y}$ $60.9 \%$ respecto al total de las viviendas pertenecientes a dichos índices.

En cambio, $54.4 \%$ del total de encuestados que residen en zonas con un grado de marginación medio reportaron la venta de sus residuos. Esto en comparación con el 36 y $31.7 \%$ del total de encuestados que habitan en áreas con grado de marginación bajo y muy bajo, respectivamente, quienes también mencionaron dicha actividad (Cuadro V). Esta situación se corroboró con las motivaciones que reportaron los S y los NS. El mayor porcentaje de encuestados que considera como una motivación el pago por los materiales reciclables vive en zonas con un grado de marginación muy alto, alto y medio. Por el contrario, el menor porcentaje motivado por la venta se encuentra en las zonas con grado de marginación muy bajo y bajo.

En lo que se refiere a la disposición a separar los

CUADRO V. VENTA DE RESIDUOS RECICLABLES SEGÚN MARGINACIÓN URBANA*

\begin{tabular}{lcc}
\hline $\begin{array}{l}\text { Marginación } \\
\text { urbana }\end{array}$ & \multicolumn{2}{c}{ Venta de residuos reciclables } \\
\cline { 2 - 3 } & $\mathrm{Si} \mathrm{( \% )}$ & No (\%) \\
\hline Muy alta & 80.0 & 20.0 \\
Alta & 60.9 & 39.1 \\
Media & 54.4 & 45.6 \\
Baja & 36.0 & 64.0 \\
Muy baja & 31.7 & 68.3 \\
\hline
\end{tabular}

Fuente: Elaboración propia

* Las respuestas no son mutuamente excluyentes, por lo que la suma es más de $100 \%$

residuos reciclables, de los 344 encuestados que respondieron a la pregunta de que si estaban dispuestos a colaborar siempre y cuando existiera un servicio de recolección de estos materiales, $93.6 \%$ de ellos $(\mathrm{n}=$ $322)$ dijeron que sí, pero de ese total $4.1 \%(n=13)$ dejaría de hacerlo si los materiales fueran recogidos por pepenadores. Por otro lado, de $6.4 \%(n=22)$ de los encuestados que dijo no estar dispuesto a separar sus residuos reciclables, $63.6 \%(\mathrm{n}=14)$ consideró que sí lo haría si los materiales fueran acopiados por pepenadores.

Por otra parte, los resultados del modelo multivariado de regresión logística binaria se muestran en el cuadro VI, indicando las variables estadísticamente significativas que aumentan la probabilidad de llevar a cabo la separación de los residuos reciclables. Se encontró que son variables predictoras de dicho hábito: a) el haber participado en alguna organización o programa relacionado al tema de los residuos o de reciclaje, b) conocer los lugares de acopio, c) estudios de nivel secundaria (versus preescolar o primaria) del(a) jefe de familia y d) tener mayor tiempo de residencia en la ciudad.

\section{DISCUSIÓN}

Los hallazgos presentados hasta ahora coinciden con resultados de diversas investigaciones, como se explica enseguida.

En materia de separación, el presente estudio encontró que $56.6 \%(\mathrm{n}=198)$ de los encuestados $(\mathrm{n}=350)$ reportó realizar la separación de residuos 
CUADRO VI. RESULTADOS DEL MODELO MULTIVARIADO DE REGRESIÓN LOGÍSTICA BINARIA

\begin{tabular}{llc}
\hline Variable & $\begin{array}{c}\text { Significancia } \\
\text { estadística }\end{array}$ & $\begin{array}{c}\text { Razón de mo- } \\
\text { mios }\end{array}$ \\
\hline $\begin{array}{l}\text { Conocimiento de problemas derivados del manejo } \\
\text { inadecuado de los residuos sólidos urbanos (RSU) }\end{array}$ & 0.537 & 0.838 \\
Conciencia sobre la importancia de la separación & 0.842 & 1.120 \\
Participación en organizaciones o programas de reciclaje & $\mathbf{0 . 0 0 7 *}$ & 2.160 \\
Interés recibir capacitación o información relacionada & & \\
al tema de los residuos & 0.574 & 0.845 \\
Conocimiento de centros o lugares de acopio & $\mathbf{0 . 0 0 0 *}$ & 4.157 \\
Género & 0.945 & 0.981 \\
Escolaridad (básica) & 0.041 & \\
Escolaridad (secundaria) & $\mathbf{0 . 0 1 6 *}$ & 2.672 \\
Escolaridad (media superior o superior) & 0.060 & 1.934 \\
Años de residencia & $\mathbf{0 . 0 2 5}$ & 1.021 \\
Edad & 0.956 & 0.999 \\
Marginación urbana (muy alta) & 0.679 & \\
Marginación urbana (alta) & 0.426 & 2.386 \\
Marginación urbana (media) & 0.466 & 2.119 \\
Marginación urbana (baja) & 0.709 & 1.474 \\
Marginación urbana (muy baja) & 0.729 & 1.445 \\
Índice socioeconómico de la vivienda & 0.400 & 0.981 \\
\hline
\end{tabular}

Fuente: Elaboración propia

* Significancia estadística: un valor menor a 0.05 indica que la variable independiente, en términos de probabilidad, explica a la variable dependiente.

La razón de momios indica la dependencia entre variables; cuanto más distante sea a 1, significa mayor fuerza de relación. Los indicadores de ajuste del modelo mostraron un $69.5 \%$ de acierto en la clasificación de los encuestados de acuerdo a la variable dependiente. La prueba de chi cuadrada comparando el modelo ajustado con un modelo nulo dio una $\mathrm{p}<0.05$, indicando que el modelo presentó una mejora significativa en la estimación de la probabilidad de ocurrencia del resultado. El valor de $\mathrm{R}^{2}$ de Nagelkerke resultó en 0.238 y la prueba de ajuste de Hosmer y Lemeshow tuvo una $p=0.939$. Los valores anteriores muestran que el modelo tiene un adecuado ajuste de los datos observados.

reciclables en 2016. En un estudio de 86 hogares de Tijuana (Luna 2002), $48.7 \%$ de los encuestados mencionó realizar algún tipo de separación de sus desechos. En 2010, de un muestreo de 120 hogares por estrato socioeconómico (alto, medio y bajo) de Tijuana, $60 \%$ de los encuestados afirmó separar sus residuos domésticos para reutilizarlos o venderlos (De la Parra et al. 2010). A nivel nacional, el Instituto Nacional de Estadística y Geografía (INEGI) reportó que en 2015 la separación de residuos en los hogares, incluyendo los orgánicos, alcanzó $57.1 \%$.

La discrepancia en los porcentajes de las distintas investigaciones (que van de 49-60\%) se debe, en parte, a que los objetivos y el tipo de preguntas para conocer este hábito fueron diferentes. Asimismo, se debe considerar que la información es un reporte verbal de los encuestados, por lo que para obtener datos más precisos es recomendable la validación de respuestas.
En la observación realizada por Luna (2002), el porcentaje de separación de residuos fue reportado considerando los desechos orgánicos e inorgánicos, a diferencia de la presente investigación donde únicamente se consideraron los residuos inorgánicos reciclables. En la investigación realizada por De la Parra et al. (2010), la separación fue reportada en función de la reutilización o la venta de los materiales reciclables, esto en contraste con el presente estudio, donde la separación se contabilizó independientemente de las razones que la explicaran.

Por su parte, el INEGI brinda información nacional, la cual hasta el momento carece de una desagregación a nivel localidad. Estas disimilitudes limitan hasta cierto punto la comparación entre los resultados, sin embargo dan una idea general respecto a los hábitos de separación en los hogares.

En lo que se refiere al tipo de residuos reciclables, en esta investigación se encontró que las viviendas 
reportaron separar en mayor cantidad el aluminio $(86.9 \%)$ y los plásticos $(85.4 \%)$; residuos que representan en la zona fronteriza $2.2 \%$ y $6 \%$ respectivamente (SEMARNAP e INEGI 1997). En Tijuana, el plástico representa al $3.71 \%$ de los desechos generados a nivel doméstico, mientras que el aluminio es equivalente al $0.43 \%$ (Luna 2002).

El tipo de residuos reciclables separados que reportan los encuestados coincide con el estudio realizado por Luna (2002), donde también se reportó que el aluminio fue el material con mayor separación. Asimismo, De la Parra et al. (2010) reportaron que el aluminio presentó una separación del $87.5 \%$, seguido de las botellas de tereftalato de polietileno (PET). En 2015 a nivel nacional, el PET y otros plásticos (77.2 $\%$ ), y el aluminio ( $74.9 \%$ ) fueron los materiales con mayor porcentaje de separación (INEGI 2015).

Así pues, todos los estudios confirman que el aluminio y el plástico son de los residuos mayormente separados, situación que podría explicarse por el valor económico que tienen en el mercado del reciclaje.

Para el caso de los factores que inciden en la separación, en lo referente a las motivaciones, en este estudio se encontró que con mayor frecuencia los $\mathrm{S}$ y NS ( $81.3 \%$ y $73 \%$ respectivamente) consideran que el cuidado del ambiente es una de las razones para separar sus residuos, lo cual coincide con las investigaciones realizadas por Vining y Ebreo (1990), y Schultz et al. (1995). Vining y Ebreo (1990) encontraron que las preocupaciones ambientales eran un común denominador entre S y NS, mientras que Schultz et al. (1995) hallaron una relación positiva entre las conductas de separación y el cuidado del ambiente.

Las motivaciones que involucran la participación y las normas sociales ("poner el ejemplo a los demás" y "que todos en mi comunidad lo hicieran"), y que fueron identificadas por los S y NS de esta investigación con $60.6 \%$ y $63.2 \%$, respectivamente, han sido referidas en el estudio de Schultz et al. (1995), donde se encontró que el reconocimiento social es un factor que motiva a los NS a iniciar prácticas de separación, que son reforzadas mediante la aprobación de la comunidad.

En este sentido, las tasas de participación en los programas de reciclaje pueden incrementarse y sostenerse conforme se establecen normas sociales como un mecanismo de presión (Schultz et al. 1995), o bien si se hace obligatoria la separación como sugiere el dato de NS en esta investigación.

Por otra parte, la segunda motivación de los NS que se refiere a conocer los lugares para llevar los residuos reciclables $(63.2 \%, \mathrm{n}=96)$ así como el resultado del modelo multivariado de regresión logís- tica binaria, que indica que existe mayor probabilidad de separar los residuos reciclables cuando se conocen los centros o lugares de acopio, coinciden con los resultados de Schultz et al. (1995), quienes hallaron que entre mayor información se tenga de los lugares de acopio, mayor es la probabilidad de separar los residuos y de participar en programas de reciclaje.

En el caso de las dificultades, se encontró que la más mencionada tanto por S y NS (18.2\% y $46.1 \%$ respectivamente) se refiere a que el camión recolector mezcla los residuos reciclables con el resto de los desechos domésticos; resultado que coincide con los datos nacionales, siendo que la principal causa para no separar los residuos en los hogares se relaciona con la mezcla de los mismos al momento de su recolección: en México esto representa una cifra de $46.6 \%$ (INEGI 2015).

Esta situación demuestra que el servicio público aún presenta deficiencias en cuanto al método de recolección de residuos. Asimismo, deja en claro que hay factores que inciden en la separación que no son propios de las viviendas, ni de sus miembros.

Las dificultades referidas a la falta de espacio (16.7\% para S y $31.6 \%$ para NS) y de botes extras (16.7\% para $\mathrm{S}$ y $41.4 \%$ para NS) han sido abordadas por Derksen y Gartrell, y De Young (citados ambos en Do Valle et al. 2004), quienes encontraron que el espacio insuficiente en las viviendas disminuye la separación de los residuos en los hogares.

Contrario a esto, en un estudio realizado en México se encontró que un mayor espacio en las viviendas es aprovechado por sus habitantes para aumentar su consumo en lugar de mejorar o adoptar los hábitos de separación (Verdugo citado en Do Valle et al. 2004); es decir, la disponibilidad de espacio por sí solo no necesariamente conduce a esta práctica. Sin embargo, sí aparece como una de las razones que justifica la no separación; en México $9.1 \%$ de los habitantes la sigue considerando (INEGI 2015).

En lo que se refiere a la venta de los residuos reciclables, en este estudio se encontró que $47.3 \%$ $(\mathrm{n}=105)$ de un $63.4 \%(\mathrm{n}=222)$ de separadores $(\mathrm{S})$ optaron por vender sus residuos; comportamiento reportado principalmente en zonas con grados de marginación urbana muy alto y alto.

La situación, en parte, está asociada a los ingresos económicos que representa esta actividad; las recompensas monetarias mostraron ser una motivación importante para los $\mathrm{S}$, más no para los NS. El $46.5 \%(n=92)$ de $\mathrm{S}$, considera que una de sus motivaciones para efectuar la separación es el pago que reciben por sus materiales, principalmente aluminio y plástico. 
En cambio, entre los NS el pago por los materiales fue la motivación menos mencionada $(38.8 \%, \mathrm{n}$ $=59$ ). Los resultados no coinciden con la revisión empírica realizada por Schultz et al. (1995), quienes concluyen que ofrecer recompensas (dinero) es más eficaz para los NS. A esta misma conclusión llegaron Vining y Ebreo (1990), quienes hallaron que los NS consideran a los incentivos económicos como un aspecto importante. No obstante, con un porcentaje de $39 \%$, los incentivos económicos sí podrían ser valiosos en una estrategia complementaria para motivar la separación entre NS en Tijuana.

Por otra parte, los resultados obtenidos en el modelo multivariado de regresión logística binaria coinciden con lo que argumentan Lybecker et al. (2013), al mencionar que los individuos que han tenido una participación previa en programas $\mathrm{u}$ organizaciones relacionadas a alguna preocupación ambiental son más partidarios a participar en políticas que incentiven el reciclaje.

De acuerdo con el modelo del presente estudio, la variable de educación secundaria (versus preescolar o primaria) incrementa la probabilidad de separar los residuos reciclables. En este caso, el resultado coincide con los estudios de Schultz et al. (1995) y Do Valle et al. (2004), donde se encontró una relación entre las variables de educación y el comportamiento de la separación, estableciendo que a mayor nivel educativo mayor probabilidad de separar los residuos.

Además de estas dos variables, también se encontró que el tiempo de residencia aumenta la probabilidad de separar los residuos reciclables. Si se parte de la premisa que las personas con mayor residencia en la ciudad tienen un mayor sentido de pertenencia, se puede establecer que las prácticas de separación pueden resultar positivas para los individuos que no son ajenos a la comunidad, tal como lo demostraron Schultz et al. (1995) en su estudio.

También es de esperarse que a mayor tiempo de residencia sea mayor el conocimiento de los centros de acopio disponibles, así como lo residuos reciclables que son recuperados o demandados en la localidad.

En resumen, la encuesta permitió identificar una serie de factores que describen los hábitos de separación de residuos reciclables reportados en las viviendas de la localidad de Tijuana. Hay patrones de respuestas que difieren entre S y NS, y también disimilitudes conforme los grados de marginación urbana. Conocer el tipo de variables que inciden en el comportamiento de los generadores de residuos ayuda a esclarecer el panorama para el establecimiento de políticas que tengan como objetivo aumentar las tasas de recuperación de los residuos reciclables.

\section{CONCLUSIONES}

La problemática de los RSU en Tijuana es compleja y requiere de la participación de actores públicos y privados. El aprovechamiento de los residuos domésticos mediante su valorización para el reciclaje es una alternativa posible. Las iniciativas que se han presentado en este tema han sido adecuadas, sin embargo por alguna u otra razón se han postergado. En este contexto, la presente investigación se enfocó a entender, mediante un método cuantitativo, las opiniones de los miembros de las viviendas respecto al manejo de sus residuos domésticos reciclables. Los resultados encontrados en este estudio pueden resumirse de la siguiente manera:

E1 $57 \%$ de los encuestados reportó separar sus residuos reciclables, sin embargo, habría que considerar la ausencia de un registro de la cantidad y regularidad del hábito de separación. También debe tomarse en cuenta el tipo de residuos separados, que en su mayoría son el plástico y el aluminio; lo cual significa una práctica focalizada hacia dichos materiales.

Los hábitos de separación se explican por factores que inciden en la misma, que van desde motivaciones y dificultades percibidas hasta condiciones sociodemográficas. Se encontró que el mayor incentivo para separadores $(\mathrm{S})$ y no separadores (NS) está relacionado con el cuidado del ambiente. El mayor obstáculo percibido por S y NS se relacionó con la logística del servicio público de RSU, específicamente con el método de recolección (mezcla de residuos).

Se encontró que existen variables explicativas para el comportamiento de separar los residuos reciclables. Aumentan la probabilidad de poner en práctica dicho hábito: la participación (actual o anterior) en alguna organización o programa relacionado al tema de los residuos o del reciclaje, el conocimiento de los lugares de acopio, los estudios de nivel secundaria (versus preescolar o primaria) del(a) jefe de familia y el tener mayor tiempo de residencia en la ciudad.

La decisión de vender los residuos disminuye conforme el IMU de las viviendas es menor, es decir, la práctica de venta se reporta más recurrentemente en zonas con carencia de servicios básicos que se asocian a menores ingresos.

Dados los resultados se pueden identificar los siguientes elementos susceptibles a considerarse en el desarrollo de estrategias, acciones, políticas y 
programas en materia de separación domiciliaria de RSU:

Las estrategias para el acopio de aluminio y plástico han sido exitosas. Sería valioso implementar estrategias similares para otros reciclables como papel y cartón, otros metales y en su caso el vidrio para ser recuperados y canalizados al mercado del reciclaje.

La conciencia ambiental y la participación en programas de reciclaje han motivado la separación de reciclables. En la medida en que se puedan reforzar estas características y actividades se podrá aumentar la participación en la separación desde los hogares.

Ofrecer un servicio de recolección separada (pública, privada o social) de residuos reciclables a las viviendas es crítico y esencial dado que existe una disposición a separar mayor al $90 \%$ y la falta de este servicio de recolección es el principal obstáculo percibido por los S y NS.

Capacitar a las personas en la separación y reciclaje, brindarles contenedores para hacerlo y difundir información de los lugares de acopio de residuos reciclables aumentarían la separación domiciliaria de los residuos entre la población.

Las estrategias deberían enfocarse hacia las personas que tienen menos tiempo de vivir en la ciudad, para así generar un mayor conocimiento de los centros de acopio disponibles y de los residuos que son recuperados o demandados en la localidad, al mismo tiempo que se genere un sentido de pertenencia a través de la educación ambiental.

En la medida en la que se generalice la práctica de separación domiciliaria de reciclables inorgánicos, ya sea por normas sociales u obligatoriedad, más personas estarían dispuestas a separar con lo que se generarían sinergias positivas con la implementación de las estrategias descritas arriba.

Estas acciones propuestas no son limitativas; son una guía para establecer medidas de mitigación a la problemática de RSU en Tijuana, situación compleja que requiere de la participación de los diversos actores públicos, privados y sociales. En este sentido, la recuperación de los residuos reciclables es una alternativa con mucho potencial en cuanto a su acopio doméstico. Identificar y abordar efectivamente los factores que motivan o dificultan la separación de los residuos a través de intervenciones desde los sectores público, social y privado, no sólo cimenta las bases para transformar a los hogares en unidades participativas, sino aumenta el éxito de los programas de reciclaje.

En suma, tener información respecto a las variables que influyen en la separación domiciliaria de los residuos reciclables es un complemento de las investigaciones que se han realizado alrededor de la
GIRSU en Tijuana. Con este estudio se dio un primer acercamiento para entender a uno de los principales generadores de residuos: los hogares, unidades de consumo que ha sido complejo incorporar a un manejo integral de los residuos.

\section{AGRADECIMIENTOS}

Este estudio fue financiado por una beca de posgrado CONACyT y un apoyo económico de El Colegio de la Frontera Norte para el desarrollo de investigación de tesis. Agradecemos al Dr. Gerardo Bernache Pérez y a la Dra. Ietza Rocío Bojórquez Chapela por su retroalimentación y recomendaciones para fortalecer el estudio, y a los habitantes encuestados por la generosidad de su tiempo. Un agradecimiento muy especial a Lina Carrascal y Marina Gurria, directoras de las organizaciones WasteLab A.C y CRECO A.C., respectivamente. Sin su apoyo esta investigación no hubiese sido posible. Finalmente, agradecemos a los dictaminadores anónimos sus valiosas observaciones y sugerencias para mejorar el manuscrito. Cualquier omisión es nuestra.

\section{REFERENCIAS}

Aprilia A., Tezuka T. y Spaargaren G. (2011). Municipal solid waste management with citizen participation: An alternative solution to waste problems in Jakarta, Indonesia. En: Zero-Carbon Energy Kyoto 2010. (T. Yao, Ed.) Springer Japan, Japón, pp. 56-62.

DOI: 10.1007/978-4-431-53910-0_7

Aragón A. (2016). Separación de los residuos sólidos inorgánicos reciclables en las viviendas de Tijuana, Baja California. Tesis de Maestría. El Colegio de la Frontera Norte A.C. Tijuana, México, 145 pp.

CDHCU (2003). Ley general para la prevención y gestión integral de residuos. Cámara de Diputados del Honorable Congreso de la Unión. Diario oficial de la federación. Ley federal. México, 53 pp.

CONAPO (2010). Índice de marginación urbana. Base de AGEB urbanas y población. Consejo Nacional de Población. Informe de resultados, México, 195 pp.

Couto I. (2008). Evaluación de la gestión integral de residuos sólidos urbanos en la frontera norte: los casos de Juárez, Reynosa y Tijuana. Tesis de Maestría. El Colegio de la Frontera Norte A.C. Tijuana, México, 229 pp.

Davies A. (2007). A wasted opportunity? Civil society and waste management in Ireland. Environ. Polit. 16 (1), 52-72. DOI: 10.1080/09644010601073564 
De la Parra Rentería C. A., Rodríguez A. L., Pacheco A. y Sánchez C. del R. (2010). Diagnóstico de los residuos sólidos urbanos en Tijuana, Baja California. El Colegio de la Frontera Norte A.C., Tijuana, México, 88 pp.

Do Valle P. O., Reis E., Menezes J. y Rebelo E. (2004). Behavioral determinants of household recycling participation: The Portuguese case. Environ. Behav. 36 (4), 505-540. DOI: 10.1177/0013916503260892

Garcés, C., Lafuente A., Pedraja M. y Rivera P. (2002). Urban waste recycling behavior: Antecedents of participation in a selective collection program. Environ. Manage. 30 (3), 378-90. DOI: 10.1007/s00267-002-2601-2

IMPLAN (2014). Mapa básico por delegaciones 2014/ Colonias 2014. Instituto Metropolitano de Planeación [en línea]. https://www.implantijuana.org/servicios/ cartografia/ 11/07/2017

INEGI (2010). Censo de población y vivienda 2010. Consulta interactiva de datos. Instituto Nacional de Estadística y Geografía [en línea]. https://www.inegi. org.mx/temas/vivienda/ 20/06/2017

INEGI (2015). Medio Ambiente. Asentamientos y actividades humanas. Datos Medio Ambiente. Instituto Nacional de Estadística y Geografía [en línea]. http:// www.beta.inegi.org.mx/datos/?t=0180000000000000 $11 / 07 / 2017$

Izazola H. (2014). Hogares y medio ambiente. Reflexiones desde la investigación sociodemográfica. En: La estadística ambiental en México. (A. M. García y C. R. López, Eds.). El Colegio de México A.C./Comisión Económica para América Latina y el Caribe, Ciudad de México, México, pp. 261-285.

Kütting G. (2004). Globalization and the environment: Moving beyond neoliberal institutionalism. International Journal of Peace Studies 9 (1), 29-46.

Luna M. C. (2002). Caracterización de los residuos sólidos domésticos y su diferenciación por estrato socioeconómico: un estudio de caso para Tijuana, B.C. Tesis de Maestría. El Colegio de la Frontera Norte A.C. Tijuana, México, $120 \mathrm{pp}$.

Lybecker D.L., McBeth M.K. y Kusko E. (2013). Trash or treasure: recycling narratives and reducing political polarisation. Environ. Polit. 22 (2), 312-332.

DOI: $10.1080 / 09644016.2012 .692935$

Manzano A.V. (1998). Selección de muestras. En: Investigar mediante encuestas. Fundamentos prácticos y aspectos teóricos. (A.J. Rojas, J. S. Fernández y C. Pérez, Eds.). Síntesis S.A., Madrid, España, pp. 51-96.

Mercado A. (2014). Datos ambientales de los hogares mexicanos. En: La estadística ambiental en México. (A. M. García y C. R. López, Eds.). El Colegio de México A.C./ Comisión Económica para América Latina y el Caribe, Ciudad de México, México, pp. 247-260.
Palatnik R., Ayalon O. y Shechter M. (2005). Household demand for waste recycling services. Environ. Manage. 35 (2), 121-129. DOI: 10.1007/s00267-004-0044-7

Palmujoki E. (2006). Public-private governance patterns and environmental sustainability. Environ. Dev. Sustain. 8 (1), 1-17. DOI: 10.1007/s10668-004-6145-x

Rodríguez A. L. (2008). Gestión local e intergubernamental de los residuos sólidos urbanos. Una evaluación de las "buenas prácticas" en los municipios mexicanos. Tesis de Maestría. El Colegio de la Frontera Norte A.C., Tijuana, México, 158 pp.

Rodríguez M. A. y Córdova A. (2006). Manual de compostaje municipal, tratamiento de residuos sólidos urbanos. Secretaría de Medio Ambiente y Recursos Naturales (SEMARNAT), Instituto Nacional de Ecología (INE), Deutsche Gesellschaft für Technische Zusammenarbeit (GTZ) GmbH, Ciudad de México, México, 102 pp.

Schultz P.W., Oskamp S. y Mainieri T. (1995). Who recycles and when? A review of personal and situational factors. J. Environ. Psychol. 15 (2), 105-121. DOI: $10.1016 / 0272-4944(95) 90019-5$

SCINCE (2012). Mapa temático. Sistema para la consulta de información censal [en línea]. http://gaia.inegi.org. $\mathrm{mx} / \mathrm{scince} 2 /$ viewer.html 17/08/2018

SEMARNAP e INEGI (1997). Estadísticas del medio ambiente. Informe de la situación general en materia de equilibrio ecológico y protección al ambiente, 1995-1996. Secretaría de Medio Ambiente, Recursos Naturales y Pesca e Instituto Nacional de Estadística y Geografía. Aguascalientes, México, 23 pp.

Shahjahan H. B. (2010). A crisis in governance: Urban solid waste management in Bangladesh. Habitat. Int. 34 (1), 125-133. DOI: 10.1016/j.habitatint.2009.08.002

UABC y MKC (2012). Waste management optimization in Tijuana, México. Universidad Autónoma de Baja California y McKinsey and Company. Diagnóstico, Tijuana, México, 56 pp.

Valderrábano M. de la L., Castro J., Hernández R. y Trujillo M. M. (2011). Las políticas públicas ambientales y su gestión en México. En: Medio ambiente, sociedad y políticas ambientales en el México contemporáneo. (E. Pérez y M. de la L. Valderrábano, Comps.). Universidad Autónoma de Guerrero/ Instituto Politécnico Nacional/ Oportunidad Renovada/ Miguel Ángel Porrúa, México, pp. 85-123.

Vining J. y Ebreo A. (1990). What makes a recycler? A comparison of recyclers and nonrecyclers. Environ. Behav. 22 (1), 55-73. DOI: 10.1177/0013916590221003 